\title{
ENSEÑANZA DE LA INTRODUCCIÓN DE LA CIENCIA DEL SUELO EN MODALIDAD A DISTANCIA EN ECUADOR
}

\section{TEACHING OF THE INTRODUCTION OF SOIL SCIENCE IN DISTANCE LEARNING IN ECUADOR}

\author{
Jiménez Álvarez, Leticia ${ }^{1}$ \\ Capa-Mora, Edwin ${ }^{1}$ \\ Quichimbo Miguitama, Pablo ${ }^{2}$ \\ Fierro Jaramillo, Natacha ${ }^{1}$ \\ 1 Universidad Técnica Particular de Loja, Ecuador. Isjimenez@utpl.edu.ec \\ 2 Universidad De Cuenca, Ecuador.
}

\section{RESUMEN}

La enseñanza de la Ciencia del Suelo a través del sistema de educación superior Modalidad Abierta y a Distancia permite, llegar prácticamente a todos los rincones del país e incluso cruzar fronteras, por ello los objetivos de este estudio fueron determinar si las barreras geográficas son limitantes para el aprendizaje de los estudiantes y evaluar la participación de los estudiantes en diversas actividades propuestas. También se consideró las actividades obligatorias propuestas y el rendimiento de los estudiantes del componente Manejo Sustentable del Suelo de la Titulación de Gestión Ambiental de todos los sectores del país, inclusive de los centros ubicados en el extranjero y se utilizaron diferentes estrategias para su aprendizaje. Los resultados del presente estudio indican que existen factores que influyen en el rendimiento de los estudiantes que van más allá de la ubicación geográfica y que la barrera territorial no es un impedimento para enseñar ésta y otras ciencias en modalidad a distancia; pero también nos muestra que las estrategias o actividades obligatorias dan mejor resultado que aquellas que son opcionales. Esos resultados nos motivan a seguir indagando sobre este tipo de educación para mejorar su calidad.

Palabras clave: Edafología; estrategias enseñanza - Aprendizaje, interacción estudiante - docente

\begin{abstract}
The teaching of Soil Science through the system of higher education, distance learning allows to reach practically all corners of the country and even cross borders, so the objective of this study was to determine if geographical barriers are limitations for the sutedent's learning and evaluate the participation of students in various proposed activities. The proposed mandatory activities were also considered and the performance of students in the subject Manejo Sustentable del Suelo of the Environmental Management Engineering career in all sectors of the country, including those located abroad. The results of the present study indicate that there are factors that influence the performance of students beyond the geographic location and that the territorial barrier is not an impediment to teach this and other sciences in distance modality; but it also shows that compulsory strategies or activities work better than those that are optional. These results motivate us to continue inquiring about this type of education to improve its quality.
\end{abstract}

Keywords: Edaphology; teaching-learning strategies, student-teacher interaction

Recibido: 01 de diciembre de 2017

Aprobado: 02 de abril de 2018

Publicado: 20 de junio de 2018

\section{Introducción}

Los temas sobre la contaminación y la disminución del suministro de recursos naturales han ganado espacio desde 1990, sin duda incluyendo a la Ciencia del Suelo como una disciplina prioritaria, debido a que el suelo mantiene e incrementa la calidad medioambiental y tiene el potencial para resolver grandes problemas de la sociedad 
(Keesstra et al., 2016; Diochon et al., 2017). Lo estudiantes en carreras relacionadas, que no necesariamente incluyen solo la agricultura tradicional. Estos cursos son requeridos en todas las Ciencias Ambientales (Havlin et al., 2010), debido a que la Edafología ayuda a comprender y resolver problemas de este tipo (Montavalli, Patton, Logan \& Frey, 2003). Como un ejemplo de la importancia del estudio recurso suelo, basta con revisar las ofertas realizadas en modalidad a distancia en programas de pregrado y posgrado.

Por ejemplo, en la Universidad de la Florida se han dictado programas como "Soil microbial ecology", "Environmental pedology" or "soil physics Laboratory", o la oferta que realizaron diversas universidades internacionales del programa "Forest and soil ecosystem services" (Curry, Grunwald \& Nair, 2007). A nivel de Latinoamérica existen experiencias interesantes: la UNAM en México en 1972, la Universidad Abierta de la Sabana en Colombia en 1975, la Universidad Estatal a Distancia en Costa Rica en 1977, la Universidad de Guadalajara (Pastor, 2005). En temas enfocados a las Ciencias Biológicas están la Universidad Central de Venezuela, que ofertó la asignatura "Química de Suelos" en modalidad semipresencial como parte del postgrado en Ciencia del Suelo (Lozano, Ruíz \& Lozano, 2015). Y en Ecuador la Universidad Técnica Particular de Loja es pionera desde 1976 en la educación a distancia, caracterizándose por presentar una amplia cobertura geográfica a través de sus centros universitarios locales, nacionales e internacionales, quienes ofertan carreras en sus cuatro áreas académicas (Biológica, Sociohumanística, Técnica y Administrativa), entre ellas la Carrera de Gestión Ambiental que incluye en su plan académico el componente "Manejo Sustentable del Suelo" (García Aretio 1999; Rubio, 2017).

En Ecuador el $40 \%$ de la población vive en áreas rurales, de quienes en su mayoría sus ingresos están direccionados a la producción agrícola y forestal (PNUMA et al., 2008). Por ello, la educación ambiental a nivel de instituciones de educación superior, ha despertado el interés de la población para poder seguir una carrera universitaria relacionada con los recursos naturales entre ellas el recurso suelo, convirtiéndose en una clara opción y alternativa para las personas con realidades diferentes, pero con el mismo deseo de superación.

En cursos a distancia un considerable contenido es impartido a través del internet (vía online), éste tipo de educación ha tenido muchas críticas por su efectividad y calidad, existiendo opiniones divididas sobre este tema. Sin embargo, es innegable las ventajas que ofrece, tal es el caso del ingreso de un gran número de personas, que por diferentes motivos no han podido continuar sus estudios, permitiendo que los estudiantes que viven en los centros rurales no tengan que recorrer grandes distancias para recibir clases o que, en muchos casos, adultos tengan que asistir a clases en una universidad en la que predominan los jóvenes (Rubio, 2017). Facilita que quienes están laborando puedan adaptarse a su tiempo y condiciones, que aquellos que desean acceder a la educación, pero por cuestiones familiares, personales, culturales y/o sociales no lo han podido hacer.

Así mismo, existen casos que los estudiantes quieren acceder a una segunda carrera, además permite el ingreso de estudiantes no tradicionales, emigrantes y/o reclusos (Bolliger \& Wasilik, 2009; García Aretio, 1999). Por otra parte, también existen barreras como los temas administrativos y sociales, habilidades académicas y técnicas, demora en la retroalimentación, convirtiendo la enseñanza online en una tarea compleja, que requiere el compromiso de todos los implicados, como son la institución, docentes y estudiantes (Bolliger \& Wasilik, 2009; Kim \& Bonk, 2006). Más aún cuando nos referimos a carreras que incluyen componentes que requieren prácticas de campo y laboratorio, en la que varios docentes al iniciar la enseñanza en cursos a distancia consideraron una tarea de difícil consecución, sin embargo de la experiencia ganada en los últimos años, se ha visto que los estudiantes de esta modalidad son más creativos para cumplir con las tareas propuestas e incluso sus reportes fueron mejores que otros estudiantes (Simonson, Smaldino, Albright \& Zvacek, 2000; Curry et al., 2007).

Según Ponce y Carrasco (2016) En el año 2014 en Ecuador el 37,1\% del área urbana y apenas el 10,4\% del área rural acceden a la educación universitaria. En cambio, el $9 \%$ de los indígenas, el 17,4 \% de los afro-negro-mulatos, el $33,4 \%$ de los mestizos y $28,5 \%$ de los blancos logran matricularse en la Universidad siendo una realidad el acceso desigual a la educación superior. Por estos antecedentes las Universidades a distancia pueden verse como una clara alternativa a quienes no pueden estudiar en las Universidades tradicionales (Casas, 2002). Pero sin alejarse de cumplir requisitos de funcionalidad, efectividad, eficiencia, disponibilidad, información e innovación (Giorgetti, Romero \& Vera, 2013) porque a pesar de existir varias experiencias a nivel de Latinoamérica y el Mundo, cada 
Universidad debe desenvolverse en su entorno y adaptarse a los cambios que el medio solicita y a las particularidades de la Carrera de las asignaturas.

Al ser la Ciencia del Suelo una disciplina transversal a otras ciencias, cuya enseñanza requiere un enfoque único (Field et al., 2011), y considerando las características propias del sistema a distancia, se requiere que los estudiantes tengan un pensamiento independiente (Field, Koppi \& McBratney, 2010), característico de los estudiantes a distancia, quienes son responsables de su educación (Driscoll, Jicha, Hunt, Tichavsky \& Thompson, 2012). El objetivo de este estudio fue determinar si las barreras geográficas son limitantes para el aprendizaje de los estudiantes de educación superior modalidad a distancia y evaluar la participación de los estudiantes en diversas actividades propuestas en la enseñanza de esta Ciencia, lo que permitirá conocer la dinámica del aprendizaje en la educación a distancia para proponer estrategias de acuerdo a las condiciones geográficas y culturales y determinar las estrategias más adecuadas para este tipo de educación.

\section{Materiales y métodos}

La asignatura "Manejo del Recurso Suelo" se dicta en la Universidad Técnica Particular de Loja, Carrera de Ingeniería en Gestión Ambiental, modalidad Abierta y a Distancia. Este tipo de educación llega a todo el país, a través de los diversos centros cantonales, ubicados en las cabeceras cantonales, que incluyen las ciudades más pobladas del país; centros provinciales ubicados en las capitales de provincia y centros internacionales, localizados en el extranjero, en países de alta migración ecuatoriana, Madrid, New York, Bolivia y Roma (Rubio, 2017).

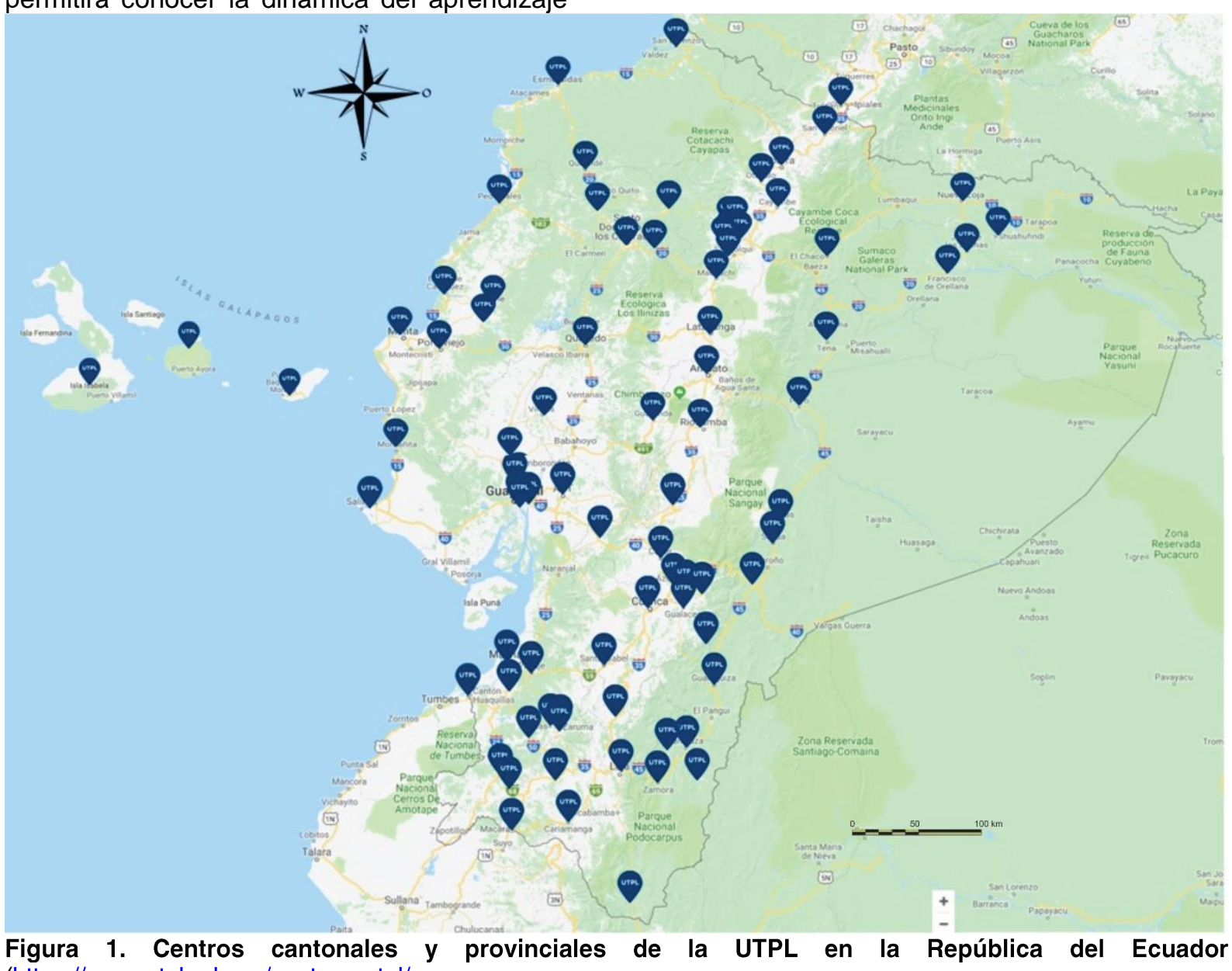

(https://www.utpl.edu.ec/centros utpl/

La materia se considera como una asignatura troncal, que proporciona la formación específica y propia de la Carrera (Rubio, 2017). Para este estudio se consideraron ocho periodos académicos, desde octubre del 2012 a agosto del 2016, cada uno de ellos tuvo una duración de aproximadamente 5 meses. Como parte del material de interacción de los estudiantes consta el texto base, que es un libro seleccionado por el docente de un autor 
reconocido que se entrega al inicio del semestre.

La guía didáctica, la misma que es previamente elaborada por uno o varios docentes que imparten el componente en la UTPL tiene como función principal es orientar, direccionar e informar. Alli se incluyen temas como datos informativos, introducción, bibliografía, orientaciones generales para el estudio, proceso de enseñanza - aprendizaje dividido en dos bimestres. En el primer bimestre se estudiaron temas relacionados a "la importancia del suelo", "Origen y evolución", propiedades físicas" y "propiedades químicas"; en el segundo bimestre las temáticas fueron "propiedades biológicas", "muestreo y análisis del suelo", "clasificación del suelo", "manejo y conservación del suelo" y "La importancia de la Edafología en la evolución y uso del territorio".

Adicional al texto y guía se envían presentaciones, vídeos, artículos e información a través de una plataforma del Entorno Virtual de Aprendizaje (EVA), constituyéndose una tecnología de comunicación imprescindible en esta modalidad de estudio. Los estudiantes tienen la opción de llamar al docente en el horario de tutorías que se da en un horario determinado por semana.

Los estudiantes de cada ciclo académico son evaluados durante dos bimestres, cada bimestre incluye un trabajo a distancia que es obligatorio y no recuperable y constituye un requisito para presentarse a la evaluación presencial, es compromiso del estudiante presentarlo en las fechas estipuladas por la UTPL. El trabajo tiene una valoración máxima de 6 puntos, de los cuales 2 puntos corresponden a preguntas objetivas 0 de opción múltiple, para ello se formularon entre 20 y 40 preguntas (se modificaron de acuerdo al ciclo). Y los cuatro puntos restantes correspondieron a las preguntas de ensayo, que en este caso consistió en una parte de campo que debía realizar el estudiante y en base a ello realizar un breve ensayo debido al enfoque práctico de este componente (Hartemink, 2015)

La evaluación es presencial y se rinde al final de cada bimestre, la misma que es valorada con hasta 14 puntos y deben rendirla en las fechas previstas por la UTPL, actividades inamovibles que no se pueden recuperar. Adicional a ello se plantearon actividades opcionales como los foros, chats y videocolaboración/cuestionario, si el estudiante contestó alguna de esas actividades, el docente calificó cada actividad sobre máximo 1 punto. La nota mínima de aprobación es 28 de 40 puntos. La valoración es A o excelente (entre 39 y 40 puntos), B o muy bueno (entre 35 y 38 puntos), C o bueno (entre 31 y 34 puntos), D o regular (28-30 puntos) y $\mathrm{E}$ o deficiente cuando obtuvieron menor a 28 puntos. Los estudiantes que obtienen menos de 28 puntos podrán rendir una evaluación de recuperación sobre 20 puntos por cada bimestre según el Reglamento de calificación modalidad a distancia de la UTPL.

\section{Análisis estadístico}

Para el análisis de datos por centro UTPL (cantonal, provincial e internacional) y género se utilizó estadística descriptiva, estos fueron expresados en porcentaje para un mejor análisis comparativo. En el caso del rendimiento se representó el promedio sobre 40 puntos de cada centro. De igual forma los datos de las actividades obligatorias y opcionales los datos fueron representados en porcentaje.

\section{Resultados y discusión}

La educación a distancia contribuye al desarrollo cultural de la población rural y urbana (Rubio, 2017), debido a que llega a los distintos sectores del país, incluso a los más alejados, con una opción clara para superar las barreras de distancia y cultura, especialmente para las mujeres, quienes en el sector rural deben ocuparse de las actividades del hogar y desempeñarse laboralmente, lo que en muchos de los casos no les da la oportunidad para inscribirse en una universidad de modalidad presencial.

En lo referente a los centros cantonales y provinciales, los resultados nos indican (Figura 2) que el porcentaje de inscritos fue similar para hombres y mujeres, a excepción del centro internacional que se observa una clara dominancia de los hombres. Los resultados de ésta investigación concuerdan con el trabajo realizado por Moncada \& Rubio (2011) en la UTPL en el periodo octubre 2009 - febrero 2010 , en donde se refleja que, de acuerdo al registro de matrícula, el $76 \%$ de los estudiantes están ubicados en el sector urbano o al menos han registrado su matrícula en un centro universitario ubicado en una capital de provincia, y únicamente el $24 \%$ pertenece al sector rural.

En Ecuador, existe un mayor número de mujeres que están accediendo a estudios superiores (Cadena \& Sánchez, 2014; Goetschel, 2014) en áreas de Administración y Comercio, Humanidades, Ciencias de la Salud y Educación, pero la realidad cambia cuando 
nos referimos a Carreras de "campo" en áreas agrícolas, pecuarias y similares o cursos de educación online relacionada a las Ciencias del Suelo, en donde se ha identificado que los porcentaje más altos están liderados por hombres (Mamo et al, 2004; Consejo Nacional de las Mujeres - CONAMU; Ministerio de Coordinación de Desarrollo Social, Unidad de Análisis e Información -SIISE; Banco Interamericano de Desarrollo - BID, 2008). Por lo que nuestros resultados son alentadores y lo tomamos como una clara opción para que las mujeres puedan desempeñarse a nivel económico, laboral y/o personal, sin abandonar sus estudios en el área de las Ciencias
Biológicas. Debido a que muchos, especialmente amas de casa requieren un trabajo a medio tiempo para subsistir y es difícil asistir en los horarios tradicionales cuando tienen una responsabilidad familiar (García Aretio, 1999; Gill \& Rengel, 2013); lo que contribuye a superar las metas de equidad. Más aún si consideramos que en el sector rural y urbano en Ecuador los ingresos promedio son más bajos para las mujeres (Quintana, 2014) limitando más aún su ingreso en la educación superior.

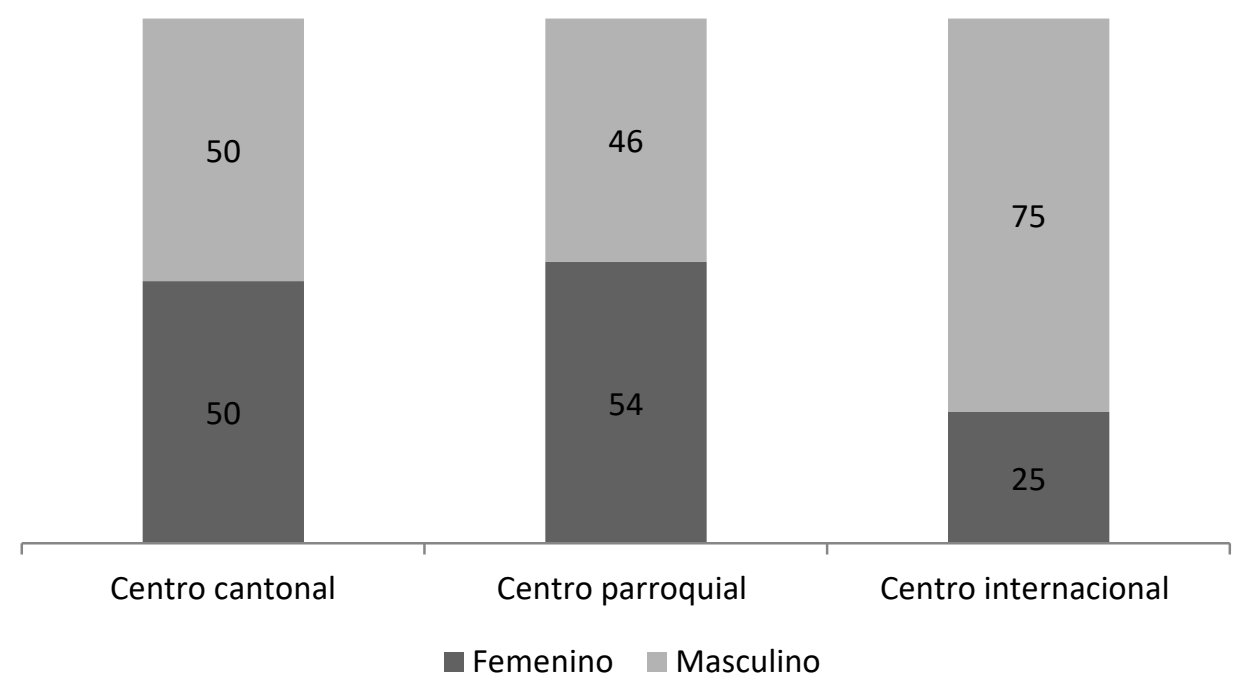

Figura 2. Porcentaje de participación por género y centro en el curso online

No se debe olvidar la relevancia personal, experiencia y contextos difieren entre grupos de aprendices (Field, Yates, Koppi, McBratney \& Jarret et al., 2017), como es el caso en este estudio, el grupo de estudiantes además de ser heterogéneo por las características que presentan cada centro, también son diversos en edad, presentando características particulares determinadas por la dispersión y ubicación geográfica, estado emocional, motivación, objetivos e intereses profesionales, carencia de tiempo y deserción (Rubio, 2009).

La participación de los estudiantes fue similar en los centros cantonales, provinciales e internacionales para las diversas actividades que se plantearon a lo largo de cada ciclo (Figura 3). El foro 1 que se planteó el primer bimestre y tuvo una participación entre 40 y 50 $\%$, siendo ligeramente mayor en el centro provincial frente a los otros dos centros. Los chats académicos y actividades propuestas tuvieron relativamente baja participación, con un porcentaje, menor al $30 \%$ en los tres centros. Para enviar los trabajos a distancia no fue una limitante la ubicación y/o distancia, debido a que en los tres centros el porcentaje fue muy alto, mayor al $90 \%$. De igual manera se observó que prácticamente todos los estudiantes se presentaron a la evaluación presencial en los tres centros, obteniendo un porcentaje mayor al $90 \%$, posiblemente porque los estudiantes conocen las fechas de entrega desde el inicio del ciclo. Claramente el hecho de conocer que los trabajos a distancia y las evaluaciones no son recuperables y tienen el mayor porcentaje de la nota, motivó a los mismos a cumplir con esta actividad, esto podría darse porque el trabajo y la evaluación 
son sólo dos veces en el ciclo, e independiente del sector de donde son los estudiantes conocían las fechas establecidas previamente por la Universidad. A diferencia de las actividades opcionales de bajo puntaje que no motivó en gran medida a los estudiantes a contestarlos, para estas actividades las fechas se comunican con aproximadamente una semana de anticipación, el tiempo podría ser insuficiente para quienes no revisan diariamente o semanalmente el correo, lo que limitó a los estudiantes en su participación, probablemente porque seleccionaron esta modalidad de estudios por las múltiples actividades que deben realizar.

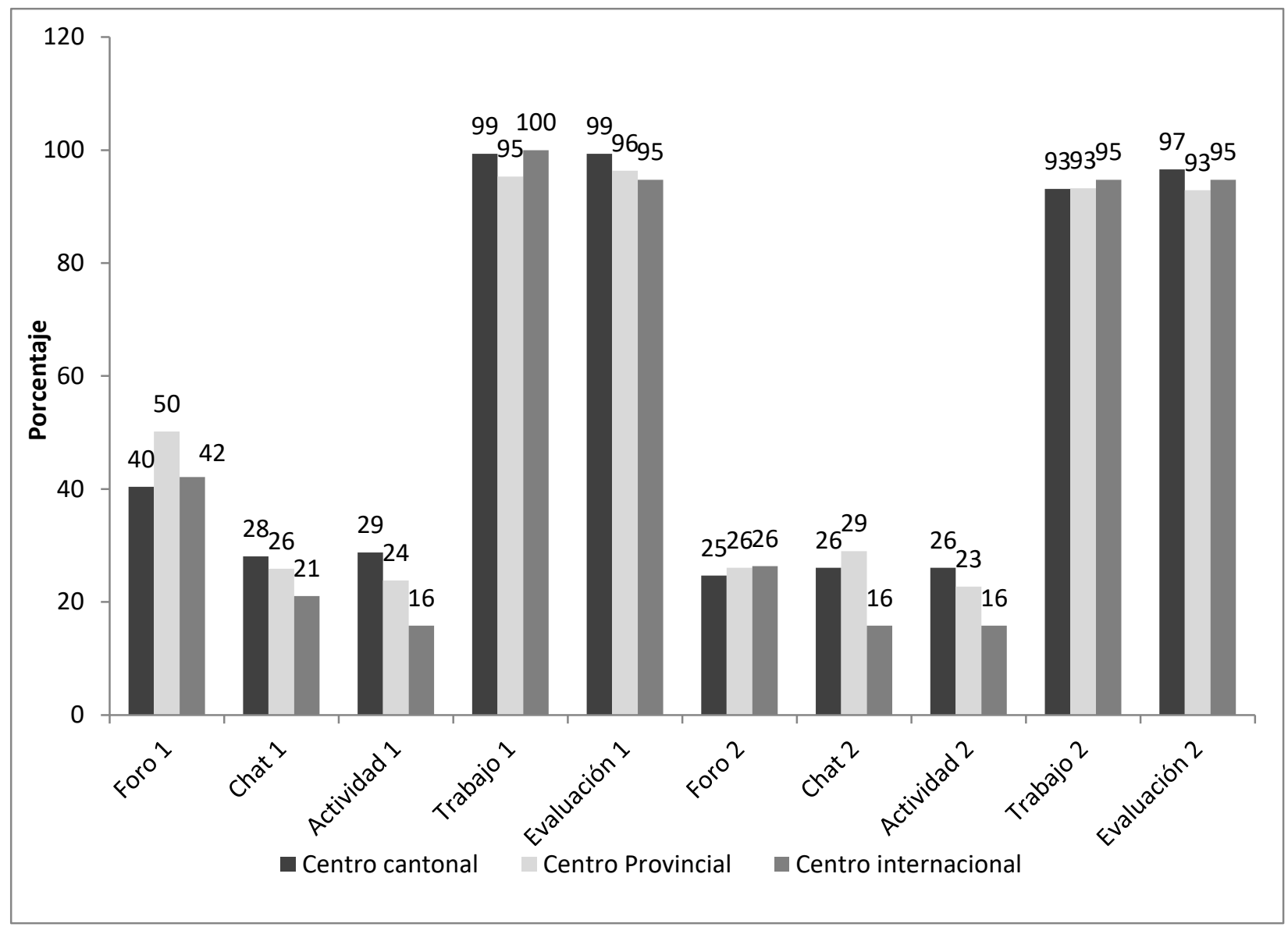

Figura 3. Porcentaje de participación en las actividades propuestas en el curso por centro. Cada barra representa el porcentaje de participación del grupo correspondiente.

El porcentaje de deserción fue bajo, probablemente porque los estudiantes de MAD vienen ya con una motivación y objetivos claros, que es la de aprobar una asignatura que permita avanzar con su carrera, por lo que la inspiración que reciba del profesor será muy importante para no abandonar el curso y concluir con la asignatura. A diferencia de la enseñanza a través de los MOOCs que, aunque se da también online y son gratuitos presentan porcentajes de reprobación muy altos (Rai \& Chunrao, 2016), probablemente porque no es parte de un Carrera y porque podrían tomarlos por diversas ocasiones hasta aprobarlo, lo que nos brinda una aproximación que la inspiración y compromiso del estudiante es fundamental para la aprobación de los componentes.

En muchos centros cantonales incluso provinciales aún el acceder al internet es una limitante, por lo que es necesario considerar a quienes por razones geográficas no disponen de este recurso en forma permanente (Alarcón, 2012). Torres (2002) señala en su diagnóstico de la educación a distancia en Ecuador, que el principal problema es el costo y la calidad de los servicios de internet que pese a que su estudio fue publicado hace más de 15 años, la realidad en torno a este tema no ha cambiado mucho en algunas zonas del país. Pero los resultados nos muestran que a pesar de la brecha digital que se presenta en la zonas rurales y urbanas y que determinan la calidad y equidad de la 
educación, porque en las zonas rurales no siempre existe servicio de electricidad e internet los estudiantes lograron cumplir con las tareas y actividades propuestas independiente del lugar de estudio (Raygada, 2003).

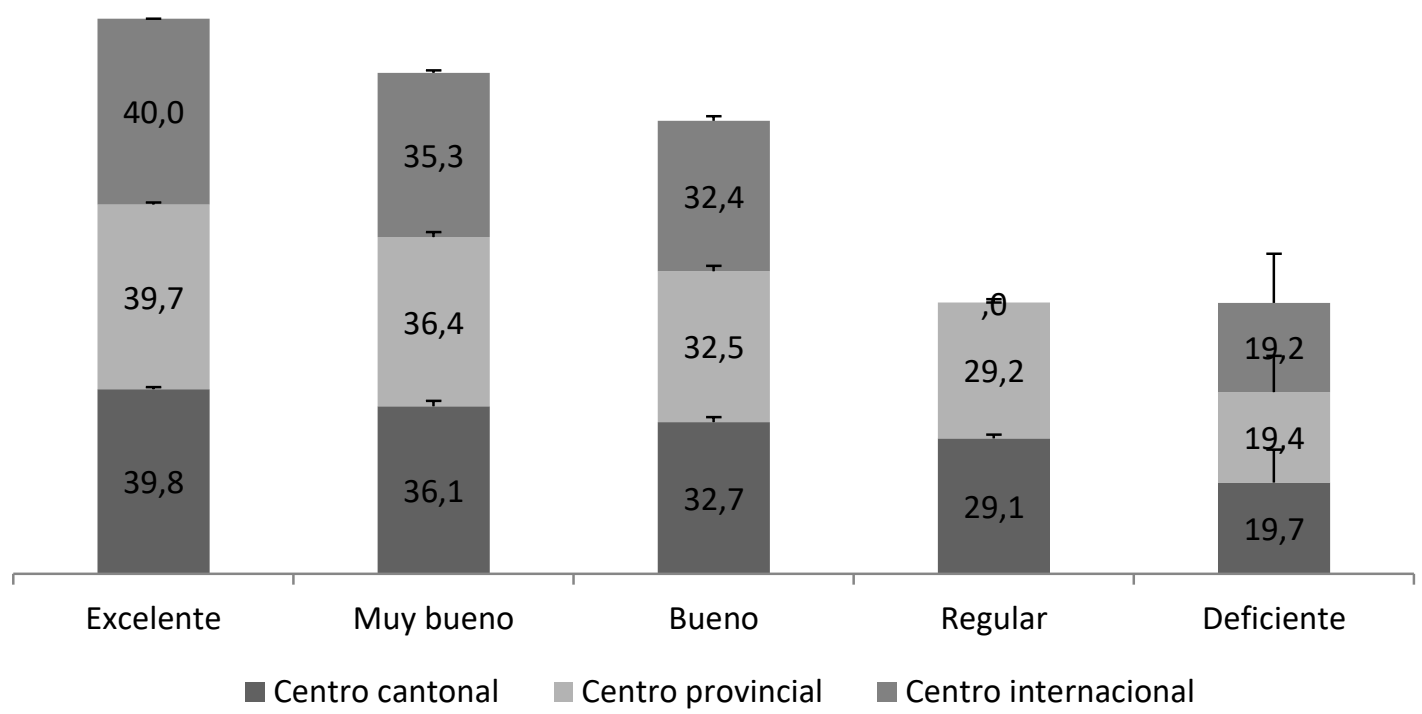

Figura 4. Promedio del rendimiento sobre 40 puntos de los estudiantes por Centro

A pesar de que en general la participación de los estudiantes fue buena y se refleja en las notas obtenidas para los tres centros (Figura 4), los autores asumimos que las calificaciones serían superiores en los centros provinciales, por las mejores condiciones que presentan estos centros, como mejor acceso al internet, mejor comunicación, el envío de material y trabajos, centros para las videoconferencias, lo que facilita el proceso de enseñanza aprendizaje. No obstante, nuestros resultados no muestran mayores diferencias, lo que contrasta con los obtenidos por Moncada \& Rubio (2011), en donde se muestra que los estudiantes que viven o se matriculan en el sector urbano sí tienen mejor rendimiento que los que lo hacen en un sector rural; aduciendo las causas a diversos factores como la calidad de la formación en el nivel secundario, el acceso a los recursos tecnológicos, ambientes laborales y sociales, así como el material complementario. Aunque para este tipo de educación la mediación es a través de integrar la tecnología en el proceso de enseñanza, debido a que expande el acceso al alumno más allá de las paredes del aula (Wade, Rasmussen \& Fox-Turnbull, 2013; Mayorga, Navas \& Pacheco, 2014), probablemente no fue el factor determinante para el rendimiento de los estudiantes. Esos resultados apoyan a lo mencionado por Savage, Birch \& Noussi (2011) que la motivación, el tener una meta y objetivos definidos ayudan al éxito y culminación de la educación a distancia. La conexión entre profesor y estudiante es una herramienta tecnológica poderosa en la educación a distancia que hace que la separación geográfica sea irrelevante (Daniel \& Lockwood, sf).

\section{Conclusiones}

Aunque los factores que influyen en el rendimiento académico de los estudiantes son diversos, y cada persona es una realidad diferente, en general en esta investigación, confirmamos que los factores geográficos no fueron una limitante para el aprendizaje de los estudiantes, pero significaron una clara opción para q las personas entre ellos mujeres puedan acceder a este tipo de Carreras en los que han dominado históricamente de los hombres.

Los factores que se puedan encontrar como determinantes principales en el rendimiento académico, deben ser correctamente trabajados por las universidades y docentes para que se puedan mejorar los resultados inmediatos de los estudiantes, y que esto se constituya en un factor que motive o influya en la permanencia de los nuevos estudiantes matriculados. Los esfuerzos que se realicen para mejorar el proceso de enseñanza aprendizaje, deberían ser de una forma divertida en la enseñanza de la Ciencia del Suelo en un sistema de educación a distancia. 


\section{Bibliografía}

Alarcón, R. (2012). La internacionalización de la educación a distancia y su alcance a las zonas excluidas dentro del territorio nacional. En M. Morocho y T. Riman (Eds.), Las nuevas fronteras de la educación a distancia, (pp. 9-17). Loja, Ecuador: Universidad Técnica Particular de Loja: CALED, Virtual Educa.

Bolliger, D. \& Wasilik, O. (2009). Factors influencing satisfaction with online teaching and learning in higher education. Distance Education, 30(1), 103-116. doi: 10.1080/01587910902845949

Cadena, F. \& Sánchez, C. (2014). Calidad y equidad en las instituciones ecuatorianas de educación superior: caminos recorridos y retos por enfrentar. En Seminario Internacional Calidad de la educación superior y género en América Latina. Quito, FLACSO 18-20 junio 2014.

Casas, M. (2002). Global and critical visions of distance universities and programs in Latin América. International Review of Research in Open and Distance Learning, 3(2). Recuperado de http://www.irrodl.org/ index.php/irrodl/article/view/111/383

Consejo Nacional de las Mujeres - CONAMU; Ministerio de Coordinación de Desarrollo Social, Unidad de Análisis e Información SIISE; Banco Interamericano de Desarrollo - BID. (2008). La situación de las mujeres ecuatorianas: una mirada desde los derechos humanos. http://www.siise.gob.ec/ siiseweb/PageWebs/pubsii/pubsii_0053.pdf

Curry, S. Grunwald, S \& Nair, V. (Eds.). (2007). Distance education, Myakka University of Florida, pp. $1-8$, https://soils.ifas.ufl. edu/media/soilsifasufledu/sws-main-site/ pdf/newsletters/fall-2007.pdf

Daniel, H. y Lockwood, P. (sf). Oz soils - an interactive introduction to soil science $« \mathrm{Oz}$ soils» - une introduction interactive à la science du sol. Symposium. 44. 1-6.

Diochon, A., Basiliko, N., Krzic, M., Yates, T.T., Olson, E., Masse, J., Amiro, B. \& Kumaragamage, D. (2017). Profiling undergraduate soil science education in Canada: Status and projected trends. Canadian Journal of Soil Science, 132, 122 132. doi.org/10.1139/cjss-2016-0058

Driscoll, A., Jicha, K., Hunt, A.N., Tichavsky, L. \& Thompson, G. (2012). Can online courses deliver in-class results? A comparison of student perfomance and satisfaction in an online versus a face-to-face introductory sociology course. American Sociological Association, 40(4), 312-331.

Field, D., Koppi, T. \& McBratney, A. (2010). Producing the thinking soil scientist. $19^{\text {th }}$ World Congress of Soil Science. 1 - 6 august, Brisbane, Australia. 1 - 4.

Field, D., Koppi, A., Jarrett, L., Abbott, L., Cattle, S., Grant, C., McBratney, A., Menzies, N. \& Weatherley A. (2011). Soil Science teaching principles. Geoderma 167-168,9-14. doi.org/10.1016/j.geoderma.2011.09.017

Field, D., Yates, D., Koppi, A., McBratney A. \& Jarret, L. (2017), Framing a modern context of soil science learning and teaching. Geoderma, 289117 - 123.

García Aretio, L. (1999). Historia de la educación a distancia. RIED, 2(1) 8 - 27. doi: https://doi.org/10.5944/ried.2.1.2084

Havlin, J., Balster, N., Chapman, S., Ferris, D., Thompson, T. \& Smith, T. (2010). Trends in soil science education and employment. Soil Science Issues. 74(5), 1429- 1432. doi:10.2136/sssaj2010.0143

Gill, J.K. \& Rengel, Z (2013). Designing an online lecture in the discipline of soil science. Teaching and Learning Forum, 1 - 9.

Goetschel, A. (2014). Género, historia y educación superior. En Seminario Internacional Calidad de la educación superior y género en América Latina. Quito, FLACSO 18-20 junio 2014.

Giorgetti, C., Romero, L. \& Vera, M. (2013). Design of a specific quality assessment model for distance education. Revista de Universidad y Sociedad del Conocimiento. 10(2), 301- 315. doi.org/10.7238/rusc.v10i2. 1742

Hartemink A. 2015. On global soil science and regional solutions. Geoderma Regional, 5, 1-3.

Keesstra, S., Bouma, J., Wallinga, J, Tittonell P., Smith, P., Cerdá, A., Montanarella, L., Quinton, J., Pachepsky, Y., Van Der Putten, W., Bardgett, R., Moolenaar, S., Mol, G., Jansen, B. \& Fresco, L. (2016). The significance of soils and soil science towards realization of the United Nations Sustainable Development goals. Soil, 2, 111-128. doi.org/10.5194/soil-2-111-2016

Kim, KJ. \& Bonk, C. (2006). The future of online teaching and learning in higher education. Educause Quarterly, 4, 22 - 30. 
Lozano, Z., Ruíz, M. \& Lozano, R. (2015). Experiencias en educación a distancia en Ciencia del Suelo. Información $y$ documentación agrícola, 31, 67-72.

Mayorga, A., Navas, Y. \& Pacheco, S. (2014). Desafíos pedagógicos ante el uso de las tecnologías de la información y la comunicación en la educación superior del Ecuador. Yachana, 3(2), 126-137.

Mamo, M., Kettler, T., Husmann, D., \& McCallister, D. (2004). Assessment of an online erosion lesson as a teaching tool in introductory soil science. North American Colleges and Teachers of Agriculture, 0319, 47-52. Recuperado de https://www. nactateachers.org/attachments/article/442/ Mamo_NACTA_Sept_04_Journal.pdf

Moncada, L. F. \& Rubio, M. J. (2011). Determinantes inmediatos del rendimiento académico en los nuevos estudiantes matriculados en el sistema de educación superior a distancia del Ecuador: caso Universidad Técnica Particular de Loja. RIED. Revista Iberoamericana de Educación a Distancia, 14.

Montavalli, P., Patton, M.D., Logan R.A. \& Frey, CJ. (2003). Promoting environmental writing in undergraduate soil science programs. Journal of Natural Resources and Life Sciences Education, 32, 93-99.

Pastor, M. (2005). La educación superior a distancia en el nuevo contexto tecnológico del siglo XXI. Revista de la Educación Superior, XXXIV(4), 77 - 93.

Ponce, J. \& Carrasco, F. (2016). Acceso y equidad a la educación superior y posgrado en el Ecuador, un enfoque descriptivo. Revista Latinoamericana de Políticas y Acción Pública. 3(2), 9- 22.

PNUMA, FLACSO, Ministerio del Ambiente, BID, ECORAE, PMRC \& ESPOL (Eds.).
2008. GEO Informe sobre el estado del medio ambiente Ecuador 2008. Pp. 33 - 34.

Quintana, Y. (2014). Género y educación superior, un reto en camino. Criterios para la transversalización de género en el sistema de educación superior ecuatoriano. FLACSO 343 - 366.

Rai, L., \& Chunrao, D. (2016). Influencing factors of success and failure in Mooc and general analysis of learner behavior. International Journal of Information and Education Technology, 6(4), 262- 268. Recuperado de http://www.ijiet.org/ vol6/697-E20018.pdf

Raygada, R. (2003). La educación a distancia en Latinoamérica. http://espacio.uned.es/ fez/eserv/bibliuned:1441/n04raygada03.pdf

Rubio, M. J. (2009). Guía General de Educación a Distancia. Loja - Ecuador: EDILOJA - UTPL. Loja - Ecuador.

Rubio, M. J. (2017). Guía General de Educación a Distancia. Loja - Ecuador. EDILOJA - UTPL, Loja - Ecuador.

Savage, N., Birch, R. \& Noussi, E. (2011). Motivation of engineering students in higher education. Engineering Education, 6(2) 39 46. doi.org/10.11120/ened.2011.06020039

Simonson, M., Smaldino, M., Albright, M. \& Zvacek, S. (2000). Review of teaching and learning a distance: foundations of distance education. Language Learning \& Technology 4(1), 16-19.

Torres, J. (2002). Diagnóstico de la educación virtual en Ecuador. IESALC. 1 - 40. http://unesdoc.unesco.org/images/0014/001 404/140469s.pdf

Wade, W., Rasmussen, K. y Fox-Turnbull, W. (2013). Can technology be a transformative force in Education? Technology in Education. $\quad 57(3), \quad 162-170$. doi.org/10.1080/1045988X.2013.795790 